\title{
Numerical Study on Similarity of Plume's Infrared Radiation from Reduced Scaling Solid Rocket
}

\author{
Xiaoying Zhang and Rui Li \\ South China University of Technology, Wushan Road, No. 381, Guangzhou 510641, China \\ Correspondence should be addressed to Xiaoying Zhang; zxy1119@scut.edu.cn
}

Received 7 January 2015; Revised 22 March 2015; Accepted 22 March 2015

Academic Editor: Yogesh Jaluria

Copyright ( 2015 X. Zhang and R. Li. This is an open access article distributed under the Creative Commons Attribution License, which permits unrestricted use, distribution, and reproduction in any medium, provided the original work is properly cited.

\begin{abstract}
Similarity of plume radiation between reduced scaling solid rocket models and full scale ones in ground conditions has been taken for investigation. Flow and radiation of plume from solid rockets with scaling ratio from 0.1 to 1 have been computed. The radiative transfer equation (RTE) is solved by the finite volume method (FVM) in infrared band $2 \sim 6 \mu \mathrm{m}$. The spectral characteristics of plume gases have been calculated with the weighted-sum-of-gray-gas (WSGG) model, and those of the $\mathrm{Al}_{2} \mathrm{O}_{3}$ particles have been solved by the Mie scattering model. Our research shows that, with the decreasing scaling ratio of the rocket engine, the radiation intensity of the plume decreases with 1.5 2.5 power of the scaling ratio. The infrared radiation of the plume gases shows a strong spectral dependency, while that of the $\mathrm{Al}_{2} \mathrm{O}_{3}$ particles shows grey property. Spectral radiation intensity of the high temperature core of the solid rocket plume increases greatly in the peak absorption spectrum of plume gases. $\mathrm{Al}_{2} \mathrm{O}_{3}$ particle is the major radiation composition in the rocket plume, whose scattering coefficient is much larger than its absorption coefficient. There is good similarity between spectral variations of plumes from different scaling solid rockets. The directional plume radiation rises with the increasing azimuth angle.
\end{abstract}

\section{Introduction}

As for base heating and thermopneumatic property of the solid rocket, plume radiation has been the focus of the investigation in thermal protection design of solid rockets in past decades [1]. Much related experimental and theoretical study has been carried out to study radiation characteristics of the solid rocket plume. As a limitation of ground test, most experiment study of rocket plume radiation used reduced scaling model, and also some of the theoretical studies used scaling model. Considering the application of research results with reduced scaling models in radiation knowledge and thermal protecting design of full scale rockets, similarity between plume radiation of reduced scaling model and full scale rocket must be obtained. However, to the authors' best knowledge, there has not been experimental study about that problem published till now. Only several numerical analysis studies can be found out.

Rozanov and Lyapustin had studied a new integral form of similarity conditions to the error analysis of truncation techniques for the forward peak scattering and deduced the integral similarity method, that is, Delta-M [2]. Jun and
Wenbing had studied the self-similarity of transmittance depth and radiation energy in a cool medium, with changing time, boundary temperature, and medium density [3]. Research of Duracz and Mccormick was focused on the ratio of two similarity parameters, radiation intensity and irradiance, and the influence of the single-scattering albedo and asymmetric coefficients within weakly absorbing clouds [4]. Mitrescu and Stephens had studied a new approach for determining the scaling parameter of the radiative transfer equation and proposed that the key assumption regarding the angular dependence of the radiative field is essential [5]. Bril et al. had studied the similarities between temperature field and concentration field in the near nozzle area; this study also proposed a method for determining a nondimensional radiance intensity with the outlet parameter, radiation wavelength, and temperature gradient of absorptivity [6].

Calculation of solid rocket plume radiation is highly complicated as the RTE is a high ordered nonlinear equation with differential term and integral terms, considering the strong spectral properties of plume gases and radiative absorption and scattering of groups of $\mathrm{Al}_{2} \mathrm{O}_{3}$ particles with different 
temperatures and diameters. The plume gases consist of several polarity gases: $\mathrm{H}_{2} \mathrm{O}, \mathrm{CO}_{2}, \mathrm{CO}, \mathrm{HCL}$, and $\mathrm{OH}$, whose infrared radiative spectrum is made up with ten thousands of lines [7]. The common detailed calculating method for gas radiation uses a band model $[8,9]$ or the weighted-sum-ofgray-gas (WSGG) model [10]. The band model has a higher precision for nonhomogeneous gases but is somewhat time lasting for large volume gases and is not very suitable for solid rocket plume gases. The WSGG model always shows a compromise of precision and calculation time, which has been widely used to calculate radiation of clouds or combustion gases. As for spectral property of $\mathrm{Al}_{2} \mathrm{O}_{3}$ particles, effects of particle temperature, concentrations, and diameters are needed to be considered $[11,12]$, and the Mie scattering theory for calculating particle radiation can also be used for $\mathrm{Al}_{2} \mathrm{O}_{3}$ particles [13]. Many numerical solution methods have been developed to calculate radiation in nonhomogenous absorptive/emissive/scattering medium by solving RTE numerically, including Monte Carlo (MC) methods [14], the streaming method (SM) [15], discrete-ordinate method (DOM) [16], and finite volume methods (FVM) [17]. To improve the solution accuracy with less execution time, the FVM has often been used to calculate the spectral radiation of the high temperature two-phase plume with highly expanding volume.

To study the similarity of infrared radiation from plumes with different scaling ratio, the Trident D5 solid rocket and a series of reduced scaling models with similar geometry have been taken for investigation. CFD code has been used to compute the axial symmetric flowing field inside the rocket engine and in plume of the full scaled rocket and reduced scaling model, with same combustion chamber pressure and plume's flowing Mach number. The spectral absorption coefficient of plume gases: $\mathrm{H}_{2} \mathrm{O}, \mathrm{CO}_{2}, \mathrm{CO}, \mathrm{HCL}$, and $\mathrm{OH}$, will be computed with WSGG model. Eight groups of $\mathrm{Al}_{2} \mathrm{O}_{3}$ particles with different diameters between 2 and $16 \mu \mathrm{m}$ have been taken into consideration. The Mie scattering model has been used to compute the spectral absorption, scattering coefficient, and phase function of $\mathrm{Al}_{2} \mathrm{O}_{3}$ particles. To calculate the spectral radiance of the plume, a finite volume method has been used to solve the RTE. Integrating spectral radiance on exterior surface of plume volume in one direction will give the plume radiation intensity. Ratio of radiation intensity between reduced scaling model and full scaled one has been computed to study the similarity rules of plume radiation.

\section{Calculation of Plume Flowing Field}

The Trident D5 solid rocket used a composite propellant NEPE; there is $10 \%$ weight of $\mathrm{Al}$ in the composite propellant. Plume's flowing data of the full scaled rocket and reduced scaling models are calculated with the same input data for the rocket nozzle, pressure is $9 \mathrm{Mpa}$, and temperature is $3750 \mathrm{~K}$. Input concentrations of major species are $f_{\mathrm{OH}}=0.192, f_{\mathrm{H}_{2}}=$ $0.299, f_{\mathrm{CO}}=0.027, f_{\mathrm{CO}_{2}}=0.146$, and $f_{\mathrm{H}_{2} \mathrm{O}}=0.0008$, on the inlet of nozzle. The full scaled rocket has a nozzle with $1.55 \mathrm{~m}$ length, $0.35 \mathrm{~m}$ diameter, and 9.7 ratio of area expansion. The reduced scaling model has the same geometry but with a minimized diameter and length. To obtain the flowing data of plume, the CEA program has been adopted to compute the chemical-equilibrium compositions of the propellants and the inlet parameters.

Then, CFD code is used to compute the united flowing in rocket nozzle and plume. The time-marching method and the advection upstream splitting method (AUSM) have been used for numerical discretization of the $2 \mathrm{D}$ axis symmetric $\mathrm{N}$ $S$ equations. The $k-\varepsilon$ turbulence model is adopted to simulate blending of the plume and atmosphere. A finite rate chemical reaction model with 12 components and 17 reactions has been taken for consideration, the detailed reaction equations and related coefficients can be seen in [18]. For the $\mathrm{Al}_{2} \mathrm{O}_{3}$ particles, the Lagrangian particle trajectory model has been used to simulate exchange of energy and momentum between particles and plume gases. The distribution of the particle diameter is determined by Braithwaite's proposed functions [19].

The plume's flowing data of the full scaled Trident D5 rocket and the 9 reduced scaling models have been calculated in our study. Only two groups of result are given in Figure 1. One is for the full scaled rocket and the other is for the 0.5 scaling model. Considering the axial symmetric characteristics of the plume's flowing field, only flowing field map on the upper $x-z$ plane has been plotted. Figure 1 shows pressure and temperature of plume gases and volume fractions of the 3 major components: $\mathrm{H}_{2} \mathrm{O}, \mathrm{CO}_{2}$, and $\mathrm{CO}$. Volume fractions of $\mathrm{HCL}$ and $\mathrm{OH}$ molecule have not been shown here as their fractions are very small, less than 0.05 . Temperature and number concentration of the 8 groups of $\mathrm{Al}_{2} \mathrm{O}_{3}$ particles have been calculated in our study, only results of 3 groups with diameters being $6 \mu \mathrm{m}, 8 \mu \mathrm{m}$, and $10 \mu \mathrm{m}$ are given here.

One can see from the figure that volume of the plume expands rapidly after the rocket nozzle, making the diameter of the high temperature core at $x=20 \mathrm{~m}$ about 5 times that of the nozzle exit. All maps of the flowing map of the 0.5 scaling rocket model have close similarity with the full scaled rocket, with similar parameter distributions and a close data range. One can also find that the high temperature areas of gases and $\mathrm{Al}_{2} \mathrm{O}_{3}$ particles are a continuous central stripe in the plume, but the dense concentration area of $\mathrm{Al}_{2} \mathrm{O}_{3}$ particles number is quite discrete. Comparing volume fraction of $\mathrm{CO}_{2}$ and $\mathrm{CO}$, it can be found that the high volume fraction area of $\mathrm{CO}_{2}$ is in the radial medium ring, at some axial distance after the nozzle exit, while that of $\mathrm{CO}$ is in the central thin stripe close behind the nozzle exit. For $\mathrm{H}_{2} \mathrm{O}$, the high volume fraction area is continuous in the central stripe of the plume.

\section{Calculation Method of Plume Radiation}

A widely used equation of radiation transport in a nonhomogeneous absorptive/emissive/scattering medium can be written as variation of radiance $i_{\lambda}^{\prime}(\overrightarrow{\mathbf{S}})$ while passing a distance $d \mathbf{S}$ along the path $\mathbf{S}$ in the medium $[20,21]$

$$
\begin{aligned}
\frac{d \mathbf{i}_{\lambda}^{\prime}(\mathbf{S})}{d \mathbf{S}}= & -\left(\Sigma \kappa_{n}+\sigma\right) \mathbf{i}_{\lambda}^{\prime}(\mathbf{S})+\left(\Sigma \kappa_{n}\right) \mathbf{i}_{\lambda, b}^{\prime} \\
& +\frac{\sigma}{4 \pi} \int_{4 \pi} \mathbf{i}_{\lambda}^{\prime}\left(\mathbf{S}^{\prime}\right) \Phi\left(\mathbf{S}, \mathbf{S}^{\prime}\right) d \omega^{\prime}
\end{aligned}
$$



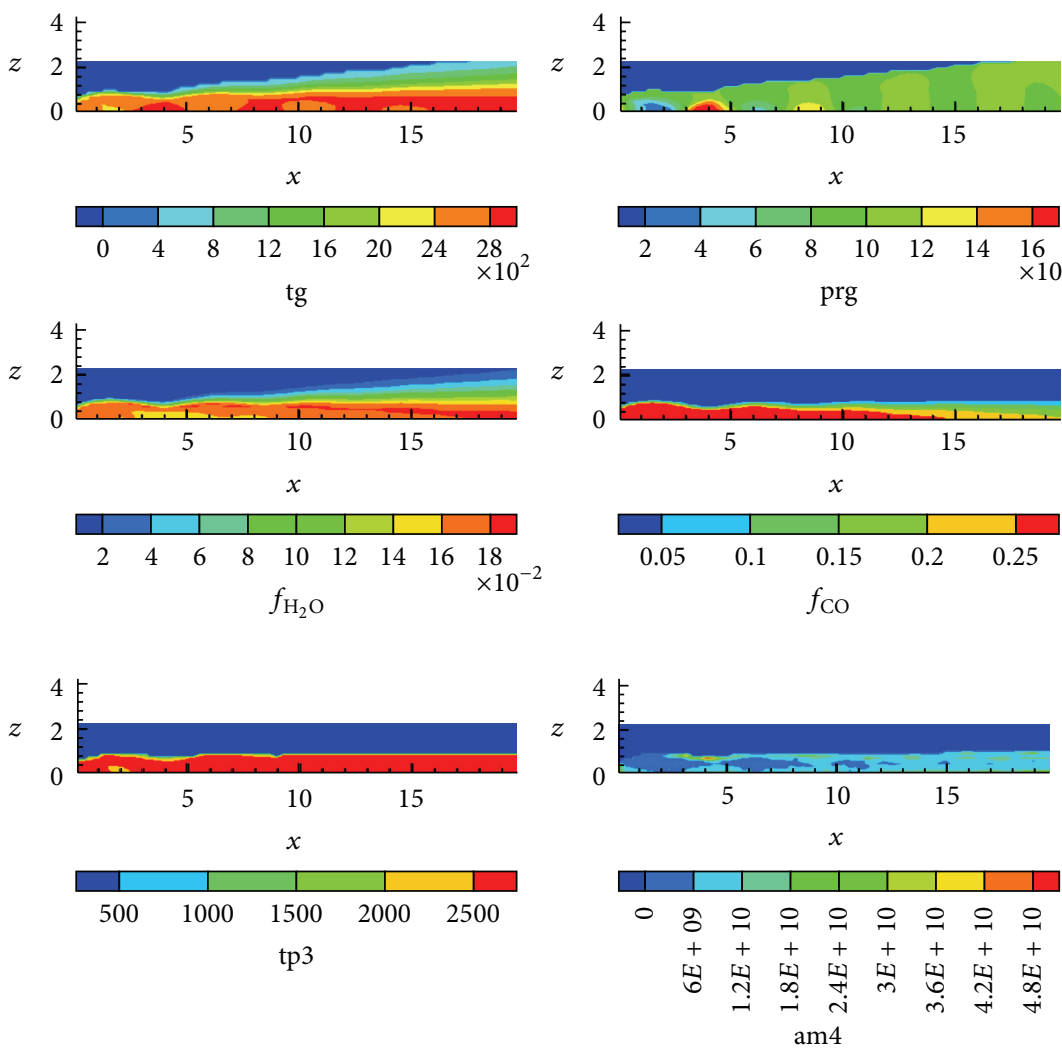

(a) Plume flowing data of 0.5 scaling sized model
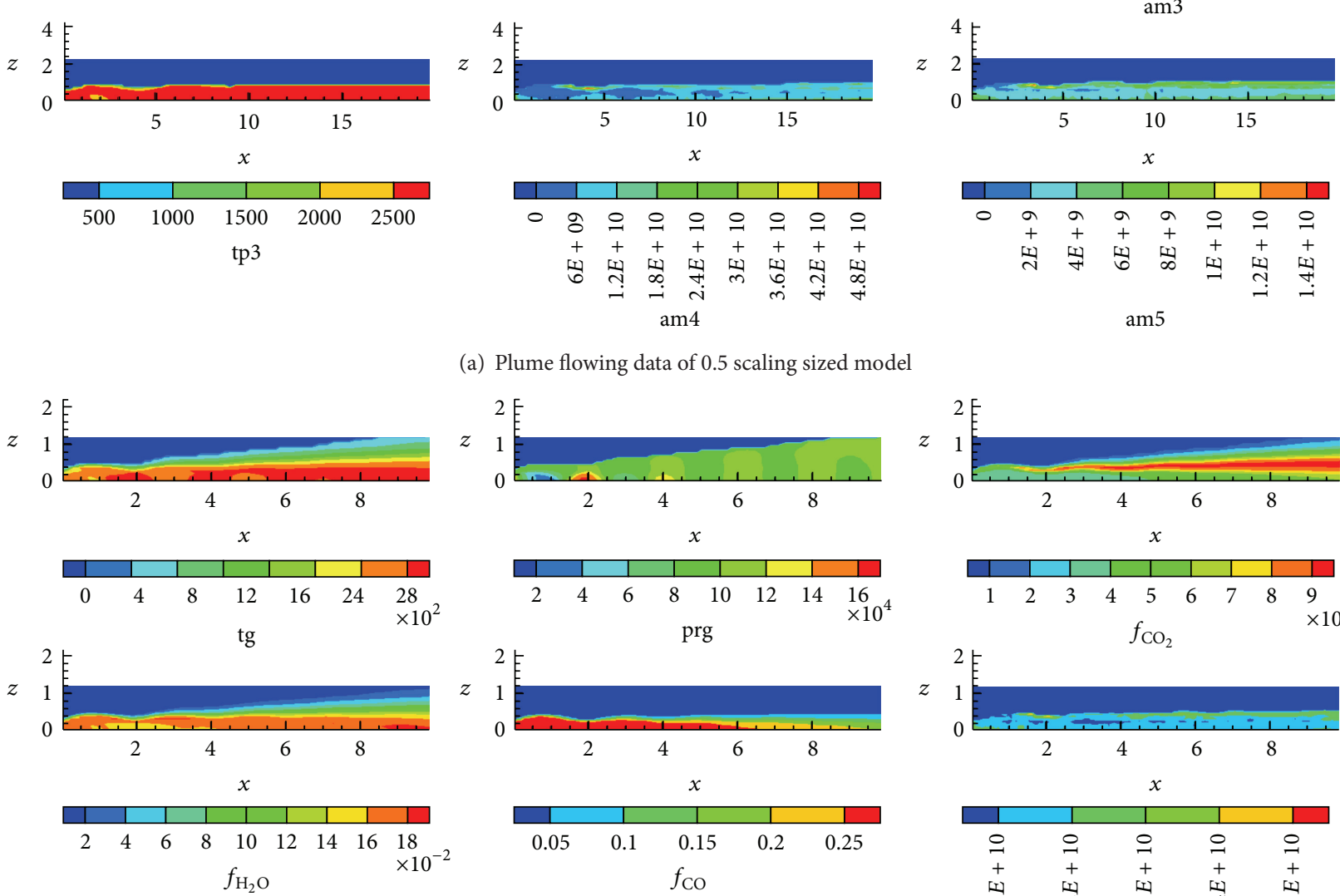

am5
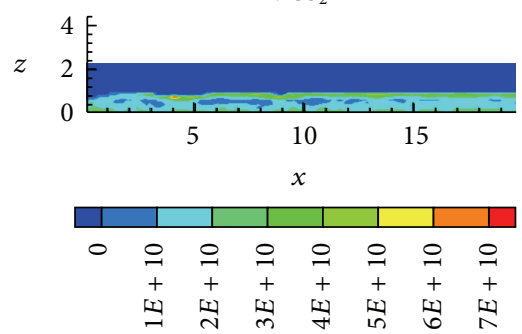
am3
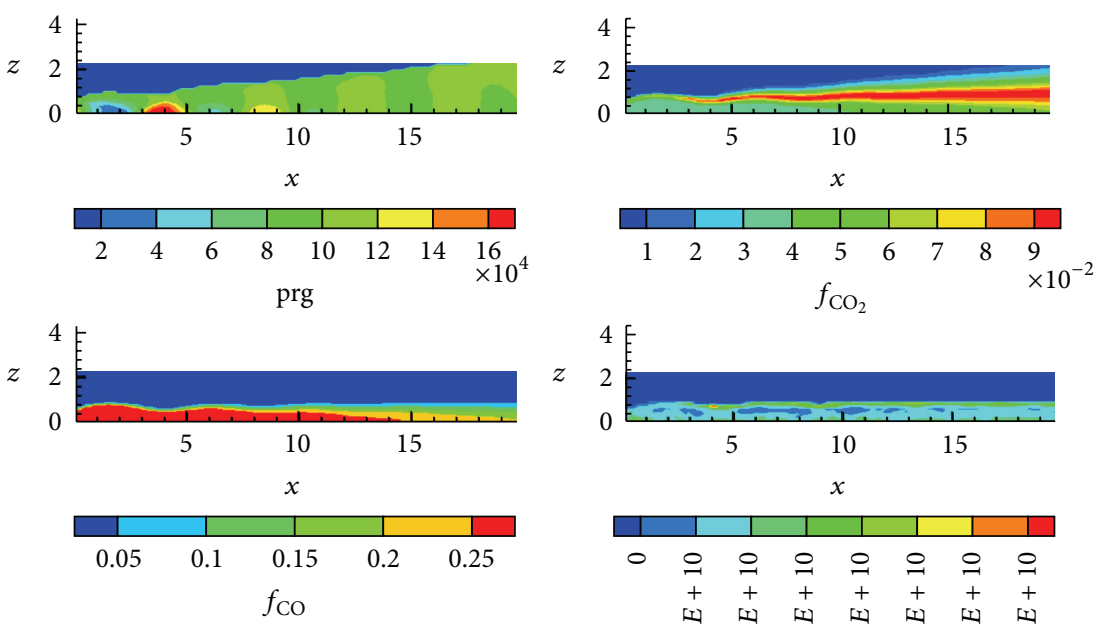


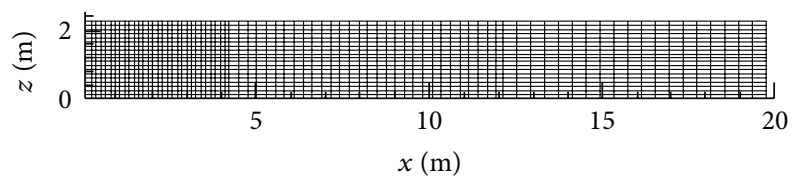

FIGURE 2: Grids for radiance calculation in the plume.

where the subscript " $\lambda$ " means spectral variable and the superscript "I" means directional variable. The symbol $\Sigma \kappa_{n}$ is the summed absorption coefficient of plume gases and $\mathrm{Al}_{2} \mathrm{O}_{3}$ particles, and $\sigma$ is the summed scattering coefficient of 8 groups of $\mathrm{Al}_{2} \mathrm{O}_{3}$ particles, which are simply summed up mathematically. The symbol $\mathbf{S}^{\prime}$ means direction of the incident radiation, $\omega^{\prime}$ means the solid angle in $\mathbf{S}^{\prime}$ direction, and $\Phi\left(\mathbf{S}, \mathbf{S}^{\prime}\right)$ means the scattering phase function between $\mathbf{S}$ and $\mathbf{S}^{\prime}$. The above symbols: $\kappa_{n}, \sigma$, and $\Phi$ are all spectral variables, while the notations " $\lambda$ " are omitted in (1) for abbreviation.

According to finite volume method, (1) can be solved to compute spectral radiance of plume by integrating the equation on a control volume. Considering the plume geometry, the cylindrical control volumes had been firstly used for solving (1), but it is found that numerical convergence of the developed coefficient matrix is very poor, being as the area difference between the two radial opposite faces of the control volume. For that reason, the cuboid control volume has finally been chosen in our study. The new computation domain for radiation computation has the same length with the flowing domain in Figure 1, but it has a square form end with side length being equal to the outer radius of the plume. The precision of plume radiance solution is mainly affected by flowing data: temperature, volume fraction, or number concentration of plume gases and $\mathrm{Al}_{2} \mathrm{O}_{3}$ particles. Those flowing data do not change dramatically in the plume as seen in Figure 1, so the mesh size for radiance solution can be much larger than that used in CFD solution. For plume of the full scaled solid rocket, the meshing plot on lengthwise section of the computing domain is shown in Figure 2. The divided grids number is $80 \times 20$ in the upper $x-z$ plane. Flowing data of control volume inside the plume takes the arithmetic average of data on CFD grids in the volume. That of control volume outside the plume is set to zero. In that case, all control volumes outside the plume would not actually influence the result of plume radiance, but the integral RTE equation is uniform for all the control volumes, and solution convergence can be improved.

Spectral radiance of each control volume is considered in a series of directions; every direction is corresponding to a set of angle, zenith angle, and azimuth angle. The zenith angle $\theta$ is defined as the angle between the plume central line ( $x$-axis) and the direction vector, and azimuth angle $\varphi$ is defined as angle between the projected direction vectors on $x-z$ coordinate plane and the $z$ axis. For radiance on control volume $P$ in direction $\mathbf{S}^{\prime}$, integrating (1) with Gaussian integral method, radiance of $P$ can be related to radiance of its neighbored control volumes in that direction. The detailed derivation procedure can be found in [19]; only the final integration equation is given here as follows:

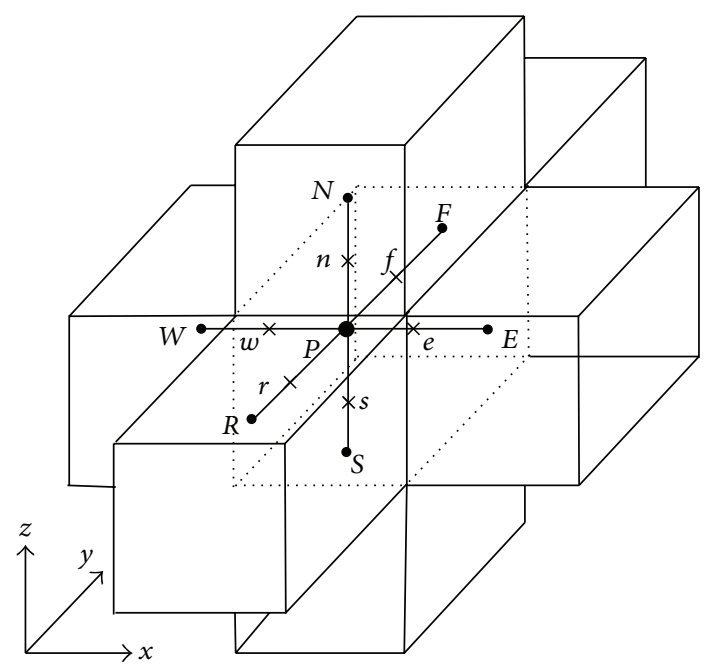

Figure 3: A control volume and the 6 neighbors.

$$
\begin{gathered}
\max \left[\Delta A_{w} D_{w}, 0\right] \mathbf{i}_{P}^{\prime}+\max \left[\Delta A_{e} D_{e}, 0\right] \mathbf{i}_{P}^{\prime} \\
+\max \left[\Delta A_{s} D_{s}, 0\right] \mathbf{i}_{P}^{\prime}+\max \left[\Delta A_{n} D_{n}, 0\right] \mathbf{i}_{P}^{\prime} \\
+\max \left[\Delta A_{r} D_{r}, 0\right] \mathbf{i}_{P}^{\prime}+\max \left[\Delta A_{f} D_{f}, 0\right] \mathbf{i}_{P}^{\prime} \\
+\left(\Sigma \kappa_{n}+\sigma\right) \Delta V_{P} \Delta \omega^{\prime} \mathbf{i}_{P}^{\prime} \\
=\max \left[-\Delta A_{w} D_{w}, 0\right] \mathbf{i}_{W}^{\prime}+\max \left[-\Delta A_{e} D_{e}, 0\right] \mathbf{i}_{E}^{\prime} \\
+\max \left[-\Delta A_{s} D_{s}, 0\right] \mathbf{i}_{S}^{\prime}+\max \left[-\Delta A_{n} D_{n}, 0\right] \mathbf{i}_{N}^{\prime} \\
+\max \left[-\Delta A_{r} D_{r}, 0\right] \mathbf{i}_{R}^{\prime}+\max \left[-\Delta A_{f} D_{f}, 0\right] \mathbf{i}_{F}^{\prime} \\
+\left[\Sigma\left(\kappa_{n}\right) \mathbf{i}_{\lambda, b}^{\prime}+\frac{\sigma}{4 \pi} \Sigma\left(\mathbf{i}_{P}^{\prime}(\mathbf{S}) \bar{\Phi}\left(\mathbf{S}, \mathbf{s}^{\prime}\right) \Delta \omega\right)\right] \Delta V_{P} \Delta \omega^{\prime} \\
\quad D_{k}=\int_{\Delta \omega^{\prime}}\left(\mathbf{S} \cdot \mathbf{n}_{k}\right) d \omega^{\prime}, \quad k=w, e, n, s, r, f \\
\bar{\Phi}\left(\mathbf{S}, \mathbf{s}^{\prime}\right)=\frac{\int_{\Delta \omega^{\prime}} \int_{\Delta \omega} \Phi\left(\mathbf{s}, \mathbf{s}^{\prime}\right) d \omega^{\prime} d \omega}{\Delta \omega^{\prime} \Delta \omega} \\
\Delta \omega^{\prime}=\int_{\varphi^{l-}}^{\varphi^{l+}} \int_{\theta^{l-}}^{\theta^{l+}} \sin \theta d \theta d \varphi,
\end{gathered}
$$

where subscripts $W, E, N, S, R$, and $F$ mean the variables of the neighbored control volume and $w, e, n, s, r$, and $f$ mean the variables on interface of neighboring volumes, as shown in Figure 3. The symbol $\Delta A_{k}$ is the area of the interface, $\mathbf{n}_{k}$ is external normal vector of interface, and $\Delta V_{P}$ is the volume of control volume.

The upper equations can be also written as the following simplified form:

$$
\begin{gathered}
a_{P} \mathbf{i}_{P}^{\prime}=a_{W} \mathbf{i}_{W}^{\prime}+a_{E} \mathbf{i}_{E}^{\prime}+a_{S} \mathbf{i}_{S}^{\prime}+a_{N} \mathbf{i}_{N}^{\prime}+a_{R} \mathbf{i}_{R}^{\prime}+a_{F} \mathbf{i}_{F}^{\prime}+\mathbf{b}_{P}, \\
a_{P}=\Sigma \max \left(\Delta A_{k} D_{k}, 0\right)
\end{gathered}
$$




$$
\begin{gathered}
+\left(\Sigma \kappa_{n}+\sigma-\frac{\sigma}{4 \pi} \bar{\Phi}\left(\mathbf{S}^{\prime}, \mathbf{S}^{\prime}\right) \Delta \omega^{\prime}\right) \Delta V_{P} \Delta \omega^{\prime}, \\
\mathbf{b}_{P}=\left[\left(\sum \kappa_{n}\right) \mathbf{i}_{\lambda, b}^{\prime}+\frac{\sigma}{4 \pi} \sum_{\mathbf{S} \neq \mathbf{S}^{\prime}} \mathbf{i}_{P}^{\prime}(\mathbf{S}) \bar{\Phi}\left(\mathbf{S}, \mathbf{S}^{\prime}\right) \Delta \omega\right] \Delta V_{P} \Delta \omega^{\prime} .
\end{gathered}
$$

The compound integral RTE equations of all control volumes have the form of vector equations

$$
[\mathbf{A}][\mathbf{I}]=[\mathbf{B}] .
$$

Equation (4) is a nonlinear system of equations, as variable $\mathbf{b}_{P}$ in the right side is actually unknown and needs to be determined by radiance of control volume $P$ in all the other directions, $\mathbf{S}^{\prime}$. So, the solution of (4) could not be completed with any iteration algorithm in one loop. A cyclic iteration algorithm with modification of $\mathbf{b}_{P}$ has been proposed in this study. In each cycle, $\mathbf{b}_{P}$ is firstly calculated with the radiance computed from the last cycle. Then, a Gauss iteration algorithm will be used to solve vector equations (4) to compute a new group of radiance for each control volume. The cyclic iteration will go on till the following convergence limitation is met:

$$
\max \left\{\mathbf{i}_{P}^{n}-\mathbf{i}_{P}^{n-1}\right\} \leq \varepsilon
$$

Considering the symmetric characteristics of geometry and flowing data of the plume, radiance of plume is also symmetric. So, we do not need to solve the vector equations of RTE on all control volumes in the plume, but only those control volumes with centers on $x-z$ coordinate plane have been chosen for calculation. While radiance of neighbored control volumes is also required in (4), those control volumes are not centering on the $x-z$ coordinate plane, and a radiance transformation algorithm on symmetric control volumes has been proposed in our study to calculate the radiance of other control volumes in the plume. The radiance transformation algorithm is derived based on rotation rules of vectors seen in Figure 4.

If the radiance of control volume $P$ in direction $S_{1}$ is $\mathbf{i}_{p}^{\prime}\left(\mathbf{S}_{1}\right)$, the radiance of control volume $O$ in direction $\mathbf{S}_{2}$ is equal to $\mathbf{i}_{P}^{\prime}\left(\mathbf{S}_{1}\right)$ when the following vector equation is satisfied:

$$
\mathbf{p}_{2}=\mathbf{S}_{2}+\mathbf{r}_{2}
$$

\section{Calculating Radiation Intensity from the Plume Surface}

In the cases of studying base heating or heat protection of the solid rocket, spectral radiation intensity on the whole surface of the plume is concerned. Spectral radiation intensity is also a directional variable, which is the integration of spectral radiance $\mathbf{i}_{\lambda}^{\prime}(\mathbf{S})$, on surface of bounding control volumes in direction S. If radiation intensity in some waveband

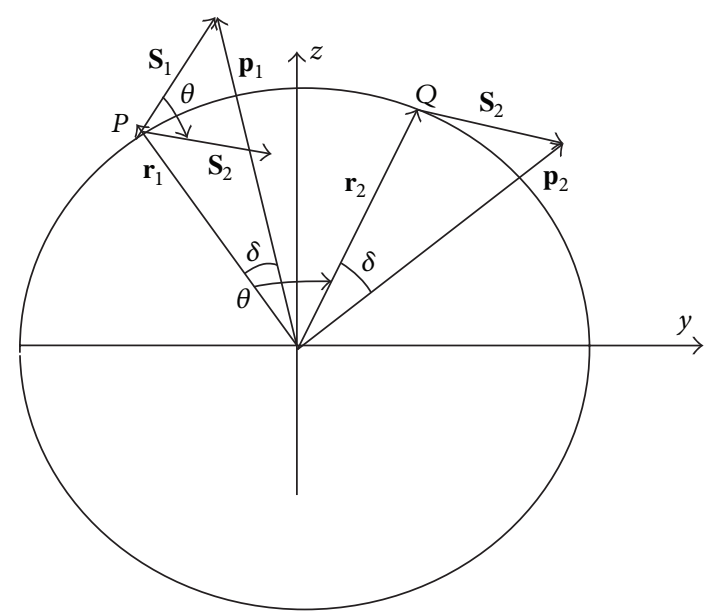

FIgURE 4: Radiance transformation on symmetric control volumes.

is concerned, as for the $2 \sim 6 \mu \mathrm{m}$ band, integration on the wavelength is also needed

$$
\begin{gathered}
I_{\lambda}=\sum_{j_{A}} \mathbf{i}_{\lambda}^{\prime}(\mathbf{S}) \mathbf{e}_{\mathbf{S}} \cdot \mathbf{n}_{\Delta A} \Delta A, \quad \mathbf{e}_{\mathbf{S}} \cdot \mathbf{n}_{\Delta A}>0, \\
I=\sum_{j_{\lambda}} \sum_{j_{A}} \mathbf{i}^{\prime}(\mathbf{S}) \mathbf{e}_{\mathbf{S}} \cdot \mathbf{n}_{\Delta A} \Delta A \Delta \lambda,
\end{gathered}
$$

where $j_{A}$ is number of control volume surfaces on boundary of the plume, $\mathbf{e}_{\mathbf{s}}$ is unit vector of the radiance direction, and $\mathbf{n}_{\Delta A}$ is unit vector of external normal on the surface.

\section{Calculation of Spectral Property of Plume Gases and $\mathrm{Al}_{2} \mathrm{O}_{3}$ Particles}

The 3 major radiation gases of the solid rocket plume are $\mathrm{H}_{2} \mathrm{O}$, $\mathrm{CO}_{2}$, and $\mathrm{CO}$. To calculate the spectral absorption coefficient of each plume gas, the standard absorption coefficient $\kappa_{\lambda \text {,STP }}$ at several temperatures and $1 \mathrm{~atm}$ pressure in $[20,21]$ has been used. For plume gas with pressure being $P$ and temperature being $T$, absorption coefficient can be modified from $\kappa_{\lambda, \text { STP }}$ as follows:

$$
\kappa_{\lambda}=\frac{P}{10^{5}} \frac{273}{T} \kappa_{\lambda, \mathrm{STP}} .
$$

Absorption coefficient of the plume gases mixture is calculated with the WSGG model, and the weight is the volume fraction of each gas. If the volume fraction of one gas is $f$, then the absorption coefficient of the gases mixture can be written with the WSGG model as

$$
\kappa_{\lambda, g}=\kappa_{\lambda, \mathrm{H}_{2} \mathrm{O}} f_{\mathrm{H}_{2} \mathrm{O}}+\kappa_{\lambda, \mathrm{CO}_{2}} f_{\mathrm{CO}_{2}}+\kappa_{\lambda, \mathrm{CO}} f_{\mathrm{CO}}
$$

The calculated absorption coefficient for plume gases $\mathrm{H}_{2} \mathrm{O}$, $\mathrm{CO}_{2}$, and $\mathrm{CO}$ and the gases mixture in center of front end of the plume have been shown in Figure 5. One can find that absorption coefficient of $\mathrm{CO}_{2}$ and $\mathrm{CO}$ is comparatively much larger than that of $\mathrm{CO}$ is $4.2 \sim 5.5 \mu \mathrm{m}$, the peak spectrum of $\mathrm{CO}_{2}$ is $4.2 \sim 5 \mu \mathrm{m}$, and absorption coefficient of $\mathrm{H}_{2} \mathrm{O}$ is much smaller. Absorption coefficient of the gases mixture appears as an accumulated effect of the three gases. 


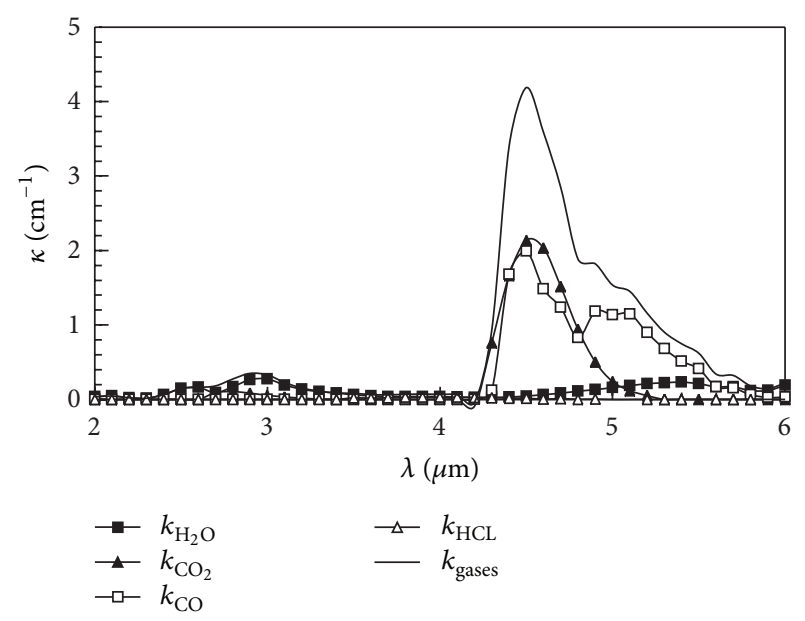

Figure 5: Calculated absorption coefficient of plume gases with WSGG model.

\section{Calculation of the Spectral Property of $\mathrm{Al}_{2} \mathrm{O}_{3}$ Particles}

In the calculation of spectral properties of $\mathrm{Al}_{2} \mathrm{O}_{3}$ particles with Mie scattering model, particle size, number concentration, and complex refractive index must be determined firstly. As mentioned above, size and number concentration of $\mathrm{Al}_{2} \mathrm{O}_{3}$ particles have been calculated in plume flowing computation. The complex refractive index of $\mathrm{Al}_{2} \mathrm{O}_{3}$ particles, $\mathbf{m}=n+\mathbf{i} k$, takes formulas recommended by Reed et al. [12]

$$
\begin{gathered}
k=4.66 \times 10^{-4} \lambda^{1.33} T^{1.5} \exp \left[-\frac{29420}{T}\right], \\
n=1.75 \cos (6 \lambda) .
\end{gathered}
$$

For a single $\mathrm{Al}_{2} \mathrm{O}_{3}$ particle, spectral properties including the scattering cross section $C_{s \lambda}$, attenuation cross section $C_{e \lambda}$, scattering factor $Q_{s \lambda}$, attenuation factor $Q_{e \lambda}$, and the scattering phase function $\Phi_{\lambda}$ can be computed with Mie scattering model [13] as

$$
\begin{aligned}
C_{s \lambda} & =\frac{\lambda^{2}}{2 \pi} \sum_{n=1}^{\infty}(2 n+1)\left[\left|a_{n}\right|^{2}+\left|b_{n}\right|^{2}\right], \\
C_{e \lambda} & =\frac{\lambda^{2}}{2 \pi} \sum_{n=1}^{\infty}(2 n+1) \operatorname{Re}\left(a_{n}+b_{n}\right), \\
Q_{s \lambda} & =\frac{2}{\chi^{2}} \sum_{n=1}^{\infty}(2 n+1)\left[\left|a_{n}\right|^{2}+\left|b_{n}\right|^{2}\right], \\
Q_{e \lambda} & =\frac{2}{\chi^{2}} \sum_{n=1}^{\infty}(2 n+1) \operatorname{Re}\left(a_{n}+b_{n}\right), \\
\Phi_{\lambda}(\theta) & =\frac{2}{Q_{s \lambda} \chi^{2}}\left[\left|S_{1}\right|^{2}+\left|S_{2}\right|^{2}\right],
\end{aligned}
$$

where $a_{n}$ and $b_{n}$ are Mie scattering coefficients and $S_{1}$ and $S_{2}$ are amplitude functions. Computation of the above

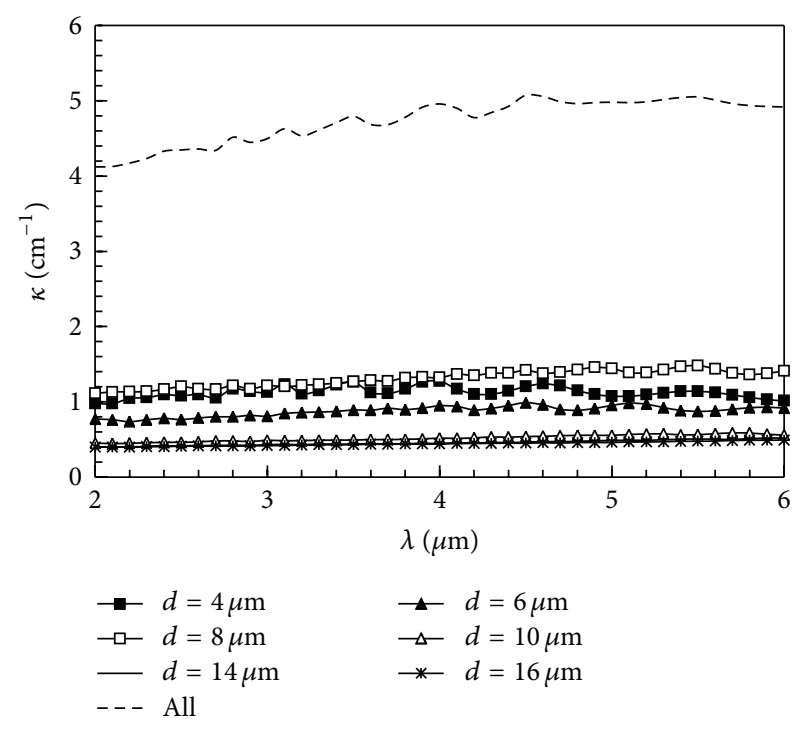

FIGURE 6: Calculated absorption coefficient of $\mathrm{Al}_{2} \mathrm{O}_{3}$ particles with Mie model.

coefficients and functions has been illustrated in detail in [13], which is not repeated in this work.

As for the spectral properties of the particle clouds, the single and independent effect of each particle is assumed and all the spectral properties of the particle clouds can be computed as the mathematic sum of all particles.

The calculated absorption coefficients of 6 groups of $\mathrm{Al}_{2} \mathrm{O}_{3}$ particles with different diameters and the total value of particles cloud in the center of plume's front end have been shown in Figure 6. That of the other two groups with $d=4 \mu \mathrm{m}, 12 \mu \mathrm{m}$ is not included in the figure as their concentration is zero at front end of the plume. One can see that absorption coefficient of $\mathrm{Al}_{2} \mathrm{O}_{3}$ particles does not change significantly with wavelength like that of plume gases. The three groups of $\mathrm{Al}_{2} \mathrm{O}_{3}$ particles with smaller diameters ( $d=4 \mu \mathrm{m}, 6 \mu \mathrm{m}, 8 \mu \mathrm{m}$ ) have larger absorption coefficient. Compared with Figure 5, it is found that the absorption coefficient of particles cloud is much larger than that of plume gases, so $\mathrm{Al}_{2} \mathrm{O}_{3}$ particles are the major radiation composition in the rocket plume.

The calculated scattering coefficients of the 6 groups of $\mathrm{Al}_{2} \mathrm{O}_{3}$ particles with different diameters and total value of particles cloud in the center of plume's front face have been shown in Figure 7. One can find that scattering coefficient for $\mathrm{Al}_{2} \mathrm{O}_{3}$ particles with $d=4 \mu \mathrm{m}$ is most strong, which is medium for particles with $d=6 \mu \mathrm{m}$ and is very small for extra particles. Compared with Figure 6, it is found that scattering coefficient of particles cloud is much bigger than the absorption coefficient, and the scattering coefficient will grow up with the increasing wavelength.

The calculated scattering phase functions of $\mathrm{Al}_{2} \mathrm{O}_{3}$ particles cloud for different wavelengths in the center of plume's front end have been shown in Figure 8. The scattering angle $\theta$ in the figure means the angle between the incident radiance and scattering direction. It can be seen that the scattering phase function in forward directions is much larger than 


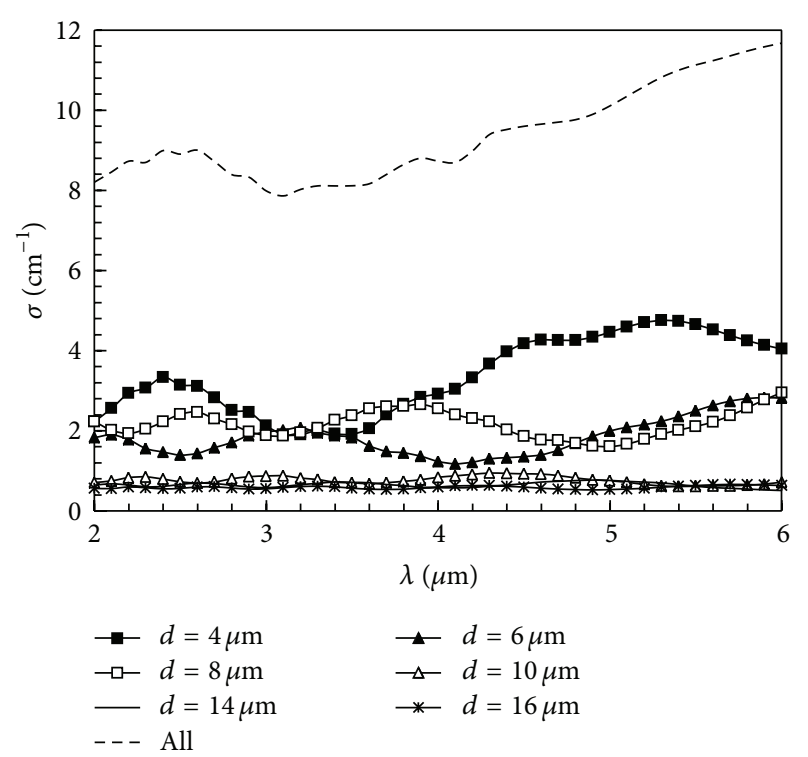

Figure 7: Calculated scattering coefficient of $\mathrm{Al}_{2} \mathrm{O}_{3}$ particles with Mie model.

that in rear directions, and scattering phase function in straight forward direction is the largest among all the five wavelengths. Comparing the results for the five wavelengths in straight forward direction, the smaller wavelength has a larger forward scattering phase function.

\section{Results and Discussion}

The calculation method of spectral property of plume gases and $\mathrm{Al}_{2} \mathrm{O}_{3}$ particles and infrared radiation intensity has been verified with a reduced scaling solid rocket in a vacuum chamber. Infrared radiation intensity in wavebands 2.7 $2.95 \mu \mathrm{m}$ and $4.2 \sim 4.45 \mu \mathrm{m}$ at three zenith angles $\theta=60^{\circ}$, $90^{\circ}$, and $120^{\circ}$ have been calculated and compared with test results. The maximum difference between calculated and test results is $20.9 \%$, which arrives at $\theta=90^{\circ}$, and the minimum difference is $10.4 \%$ which arrives at $\theta=60^{\circ}$ [22].

Studying the similarities of plume's radiation intensity from solid rocket with different scale and ratio of plume radiation between the reduced scaling and full size rockets in wavelengths $\lambda=2 \mu \mathrm{m}, 3 \mu \mathrm{m}, 4 \mu \mathrm{m}$, and $5 \mu \mathrm{m}$, and waveband $2 \sim 6 \mu \mathrm{m}$ have been calculated and illustrated in Figure 9. The horizontal ordinate $r$ in the figure means the scaling ratio of rocket model, and the vertical ordinate $I / I_{0}$ means the ratio of plume's radiation intensity. The three dashed lines are corresponding to the $1.5,2$, and 2.5 power function of scaling ratio. Results of $\theta=0^{\circ}, 40^{\circ}, 80^{\circ}, 120^{\circ}$, and $160^{\circ}$, with $\varphi=30^{\circ}$, have been investigated. It can be seen in the figure that ratio of radiation intensity increases with the increasing scaling ratio, for all the four wavelengths and waveband $2 \sim 6 \mu \mathrm{m}$. The increasing rule of radiation intensity ratio for $\theta=0^{\circ}$ gets close to the curve of 1.5 power mostly, which gets closer to the 2power function of scaling ratio for $\theta=40^{\circ}$ and comes up to reach 2.5 power of scaling ratio for $\theta=120^{\circ}$ and $160^{\circ}$. This is caused by changing the length of high radiance section and

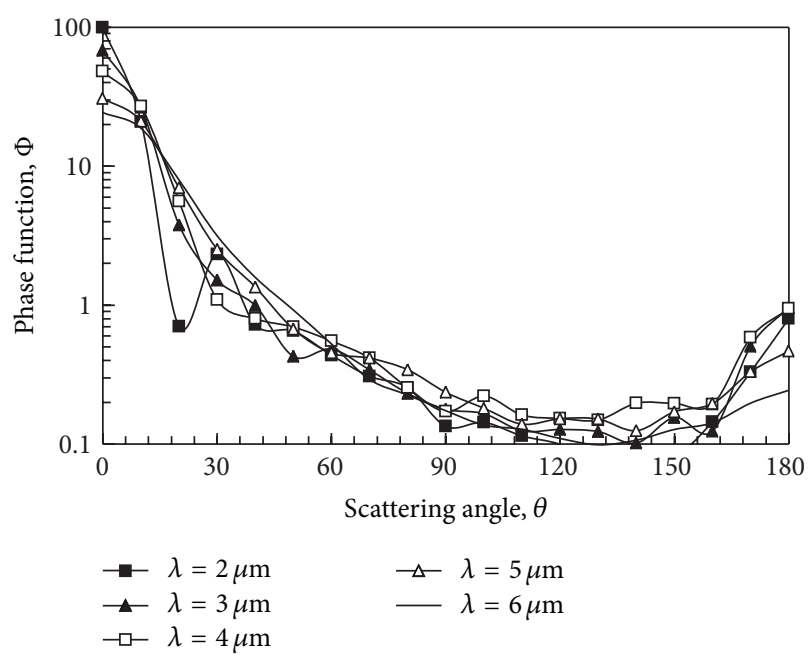

Figure 8: Calculated scattering phase function of $\mathrm{Al}_{2} \mathrm{O}_{3}$ particles with Mie model.

radiance attenuation of the cold gases in tail section of the plume. As plume's radiance mainly comes from the central stripe which has a higher temperature and concentration for both gases and $\mathrm{Al}_{2} \mathrm{O}_{3}$ particles. Radiance attenuation of the tail cold gases increases more rapidly than the projected area on the surface of the plume with the growth of the scaling ratio, making growing rate of $I / I_{0}$ being smaller than that of surface area; the latter has a growing rate of $r^{2}$ in small zenith angle. With the increase of zenith angle, the effect of radiance attenuation decreases greatly and causes the ratio of radiation intensity increasing to reach and surpass $r^{2}$.

In the viewing of spectral characteristics for solid rocket plume with different scaling ratios, the variation of radiation intensity with wavelength for the 9 reduced scaling rocket models and the full scaled one in two directions, $\theta=$ $30^{\circ}$ and $90^{\circ}$ with $\varphi=30^{\circ}$, has been plotted in Figure 10. One can see good similarity between spectral variations of different scaling rocket models. There are two peak spectra of radiation intensity, 2.7 3.0 $\mu \mathrm{m}$ and 4.3 4.6 $\mu \mathrm{m}$. Compared with Figure 5, it is found that the former spectrum is the feature radiative spectrum of $\mathrm{H}_{2} \mathrm{O}$; the latter is of $\mathrm{CO}_{2}$ and $\mathrm{CO}$. Besides, in the two peak spectra, spectral radiation intensity for plume of the 10 rockets decreases with the increasing wavelength in $2 \sim 6 \mu \mathrm{m}$ waveband. Since the $\mathrm{Al}_{2} \mathrm{O}_{3}$ particle is the major radiation composition in the plume, which has the maximum radiation for $\lambda<2.0 \mu \mathrm{m}$ as particle, the temperature is higher than $2500 \mathrm{~K}$.

The spectral radiation intensity of plume in all the zenith angles with $\varphi=30^{\circ}$, for the 0.5 scaling rocket model and the full scaled one, has been shown in Figure 11; results of wavelength $\lambda=2 \mu \mathrm{m}, 3 \mu \mathrm{m}$, and $4 \mu \mathrm{m}$ have been plotted. With the increase of the zenith angle, radiation intensity of the plume firstly increases, arrives at a maximum value in a middle angle, and decreases after that angle, since the projected area on plume surface changes with the zenith angle. Comparing radiation intensity of plume in the front and rear hemisphere, it's found that is stronger in the rear hemisphere. As read end surface of plume has a strong radiance in the rear hemisphere. 


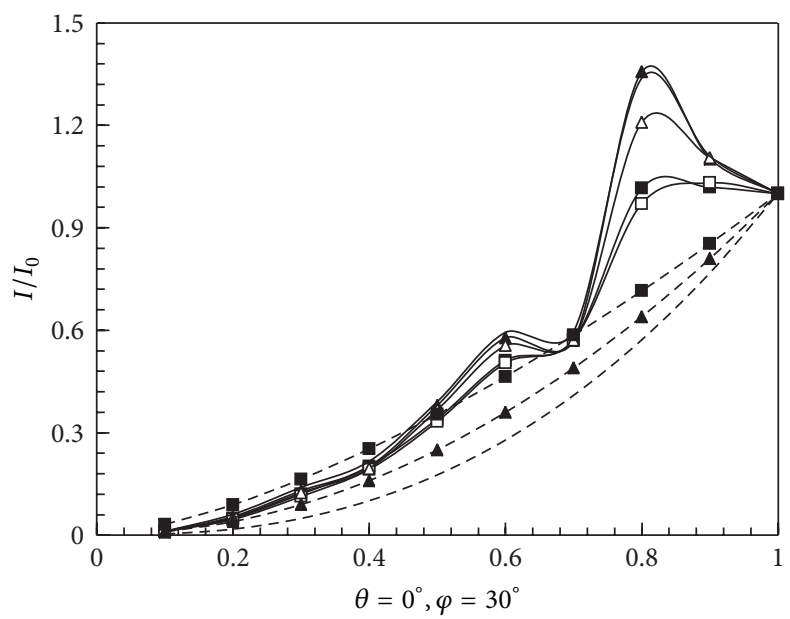

(a)

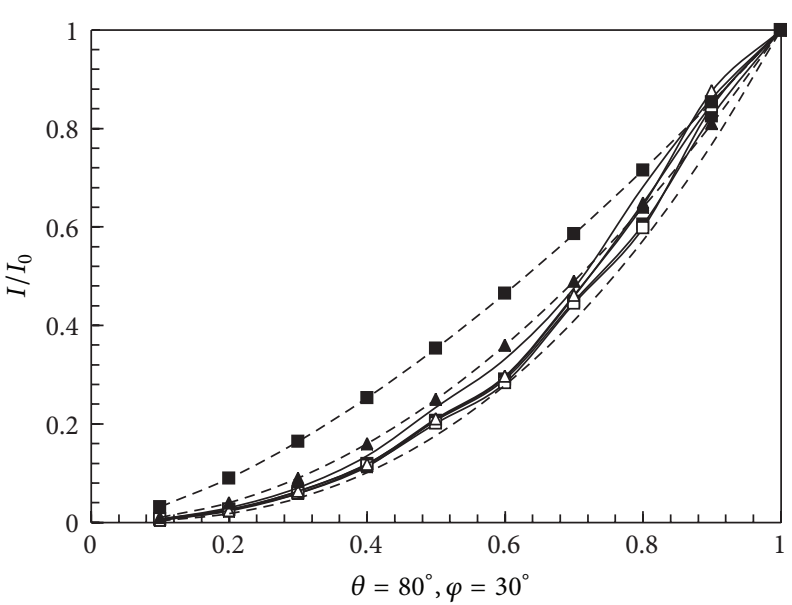

(c)

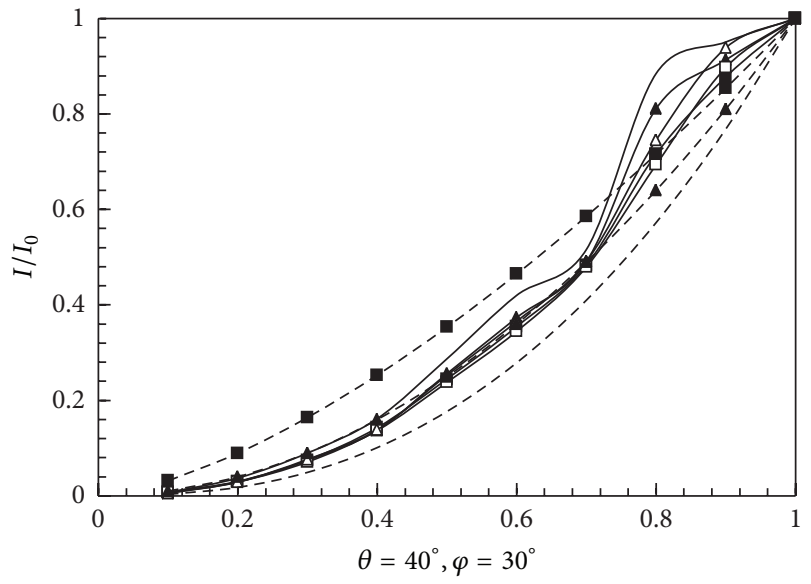

(b)

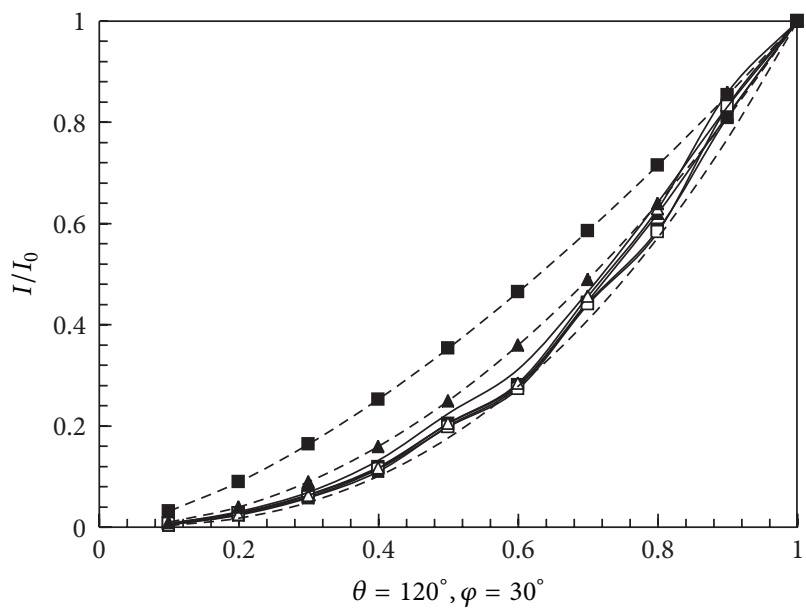

(d)

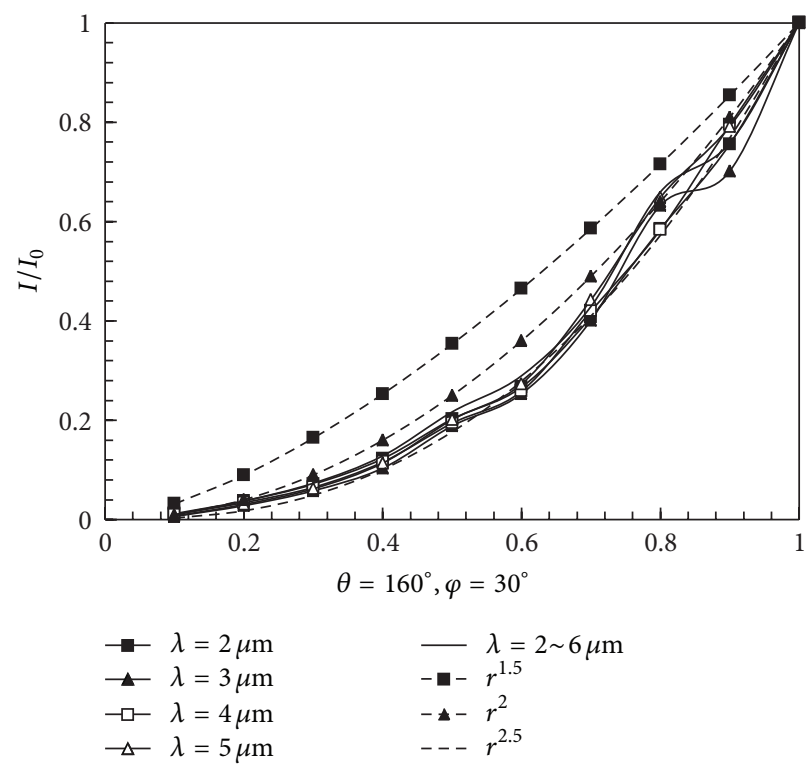

(e)

FIGURE 9: Ratio of plume radiation in the reduced scaling and full size rockets. 


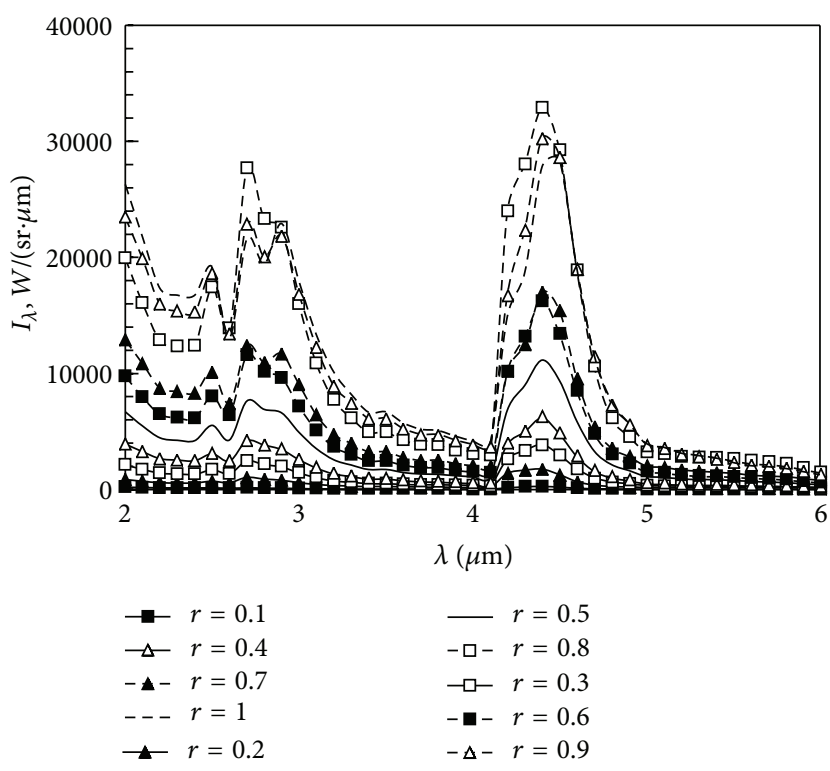

(a)

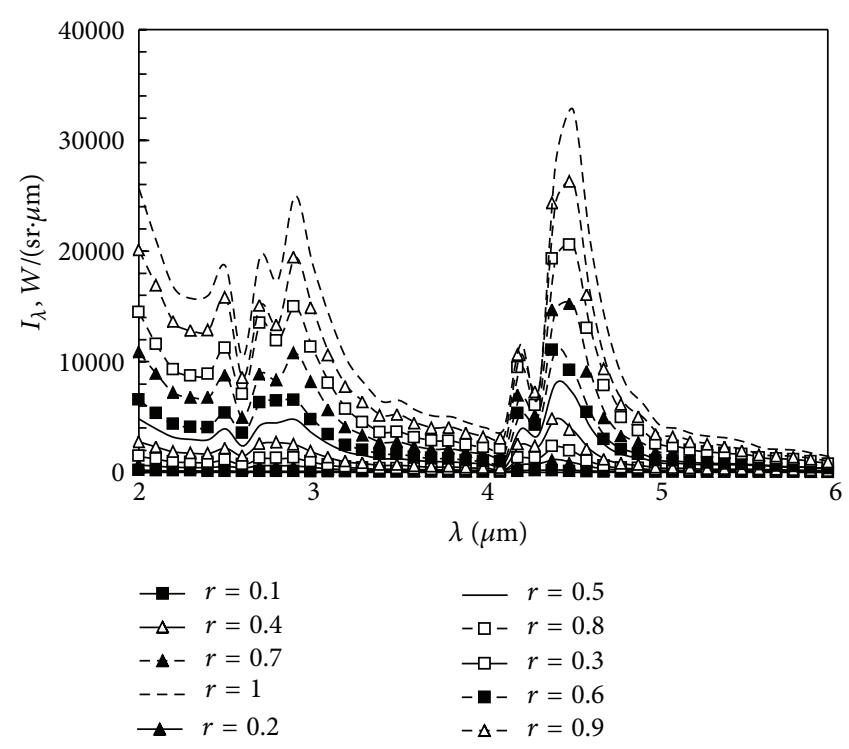

(b)

FIGURE 10: Spectral radiation intensity of plume surface.

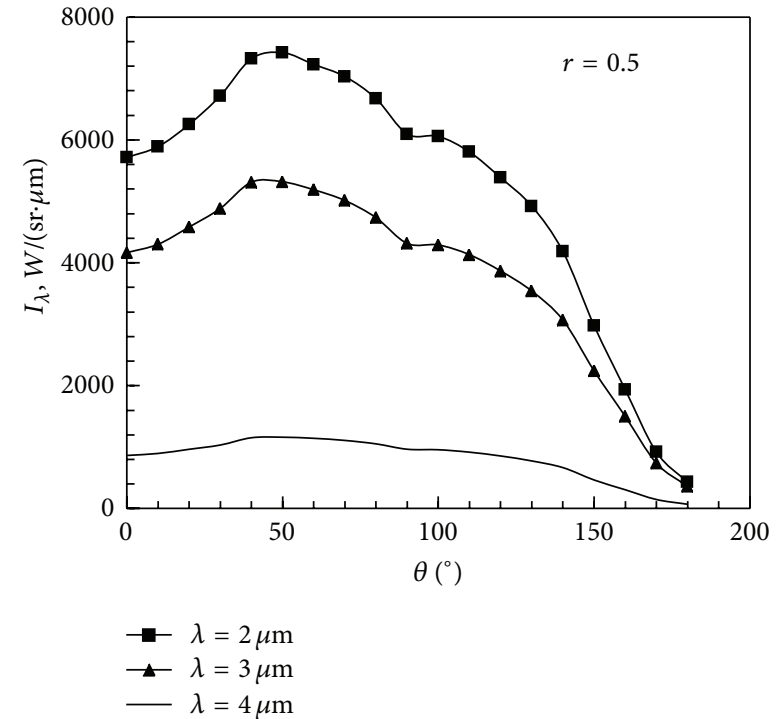

(a)

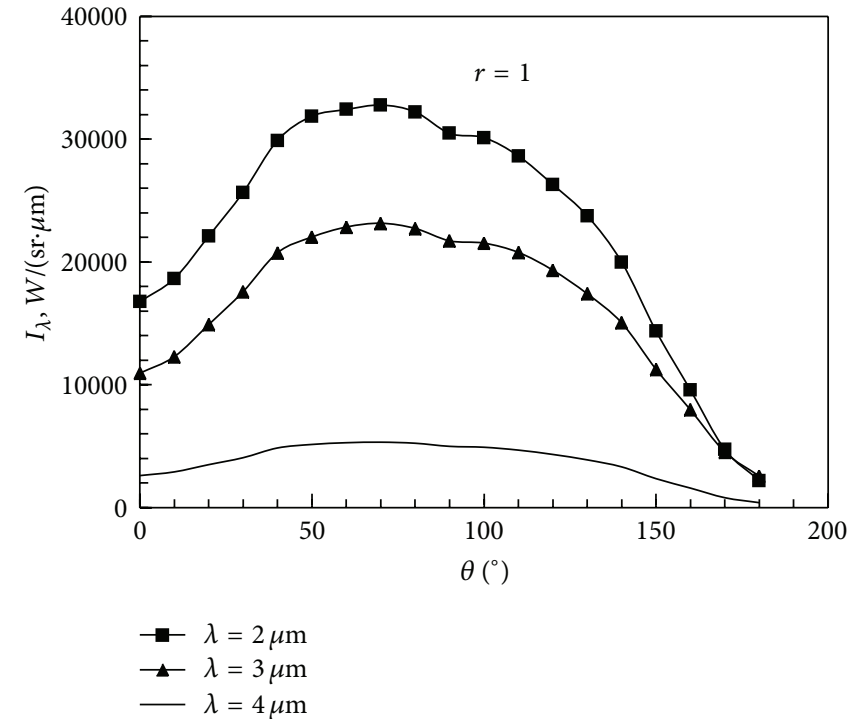

(b)

FIGURE 11: Variation of radiation intensity of plume with zenith angles.

Effect of radiance from the rear end also makes the direction with maximum radiation intensity deflects toward the rear hemisphere, which is remarkable for $r=0.5$, and the maximum radiation intensity arrives at $\theta=50^{\circ}$ in that case. The colder section in the tail of plume is shorter and the radiance from the rear end surface would be bigger for a smaller scaling rocket.

Figure 12 shows map of spectral radiance on $x-z$ coordinate plane in plume flow of the 0.5 scaling solid rocket and the full scaled ones, for wavelength $\lambda=3 \mu \mathrm{m}$ and $5 \mu \mathrm{m}$, in $\theta=0^{\circ}$, $45^{\circ}$, and $90^{\circ}$. The radiance map of the two solid rockets has similar shape and close magnitude. It is also found that the high light area with a strong radiance is located in the central stripe of the plume, where temperature and concentration of gases and $\mathrm{Al}_{2} \mathrm{O}_{3}$ particles are very high. Compared the width of the high light stripe in radiance maps of the two wavelengths, it is narrower for $\lambda=3 \mu \mathrm{m}$ than $\lambda=5 \mu \mathrm{m}$. As the radiation of $\mathrm{Al}_{2} \mathrm{O}_{3}$ particles plays the main role in plume radiance of $3 \mu \mathrm{m}$, the area with dense concentration of particles is smaller in radius than plume gases, causing high radiance area centralizing in the plume in that wavelength. Plume gases are the major radiation composition for $\lambda=$ $5 \mu \mathrm{m}$, which has a bigger expansion angle and radius size, and cause a wider stripe with high radiance. Viewing the axial 

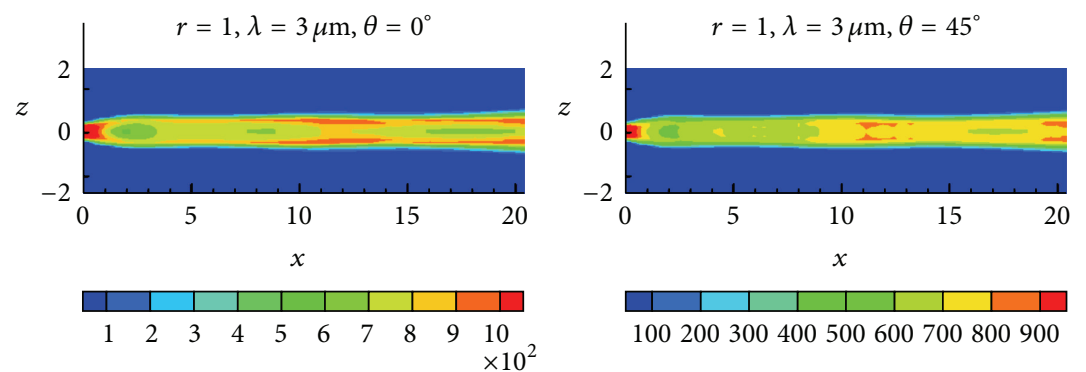

100200300400500600700800900
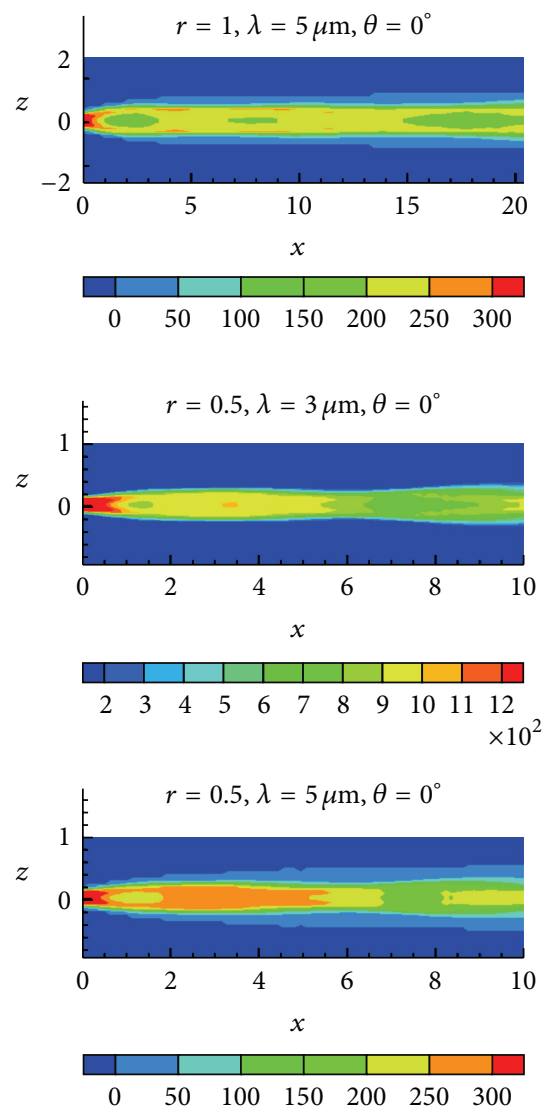
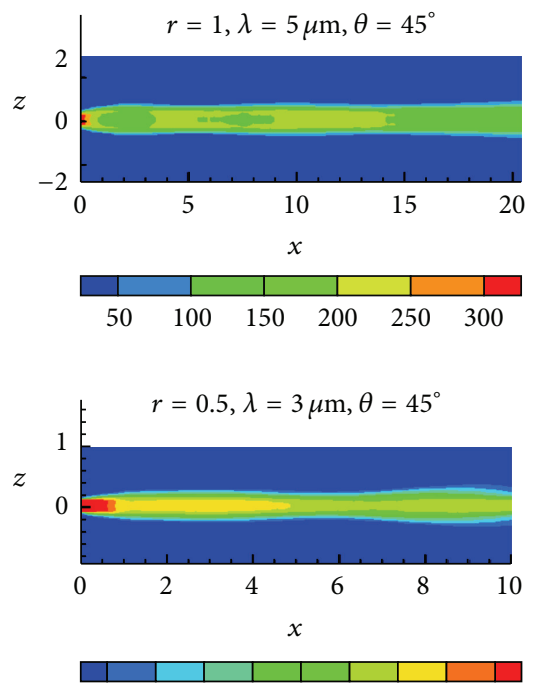

100200300400500600700800900

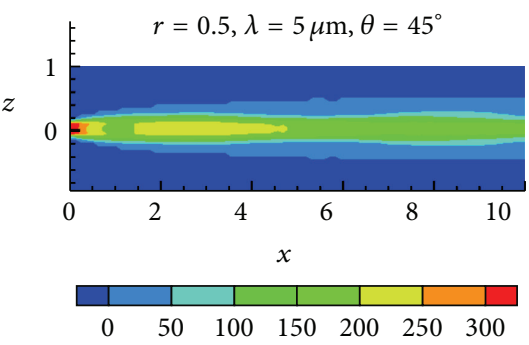

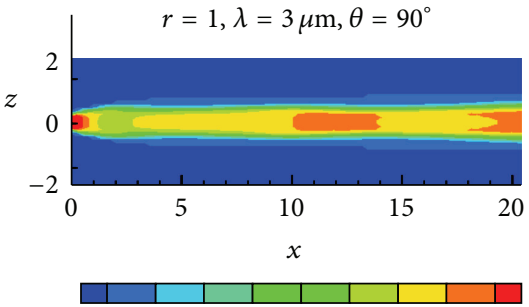

$0 \quad 100200300400500600700800$
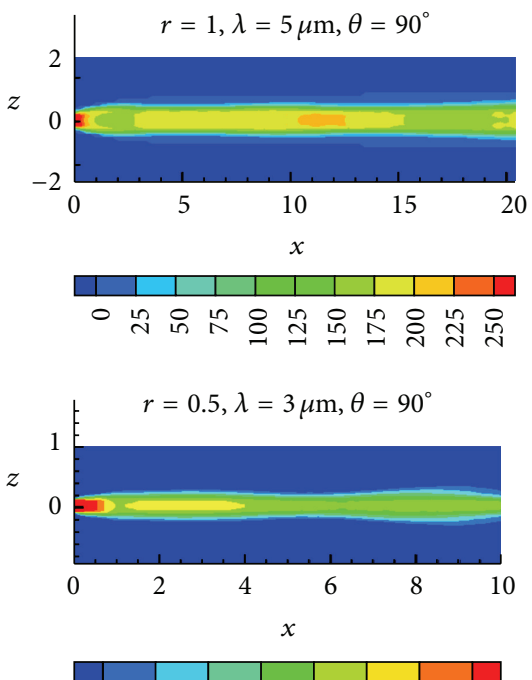

100200300400500600700800

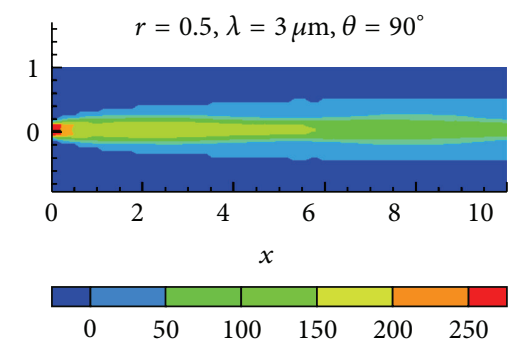

FIGURE 12: Map of spectral radiance on $x-z$ coordinate plane.

length of the high light stripe, it is in the front part with half length of the plume for the 0.5 scaling model, but extends from the front to end part of plume for the full scaled rocket model.

\section{Conclusion}

Infrared radiation of plume from one full scaled solid rocket as well as the 0.1 0.9 reducing scaled models in ground condition has been investigated to study the similarity of plume's infrared radiation for rocket models with different scaling ratios. Flowing field in the rocket and plume has been computed with CFD code, the omnidirectional and infrared radiance on symmetric plane in the plume has been calculated with the developed FVM code. The research shows that $\mathrm{Al}_{2} \mathrm{O}_{3}$ particle is the major radiation composition in the rocket plume, whose scattering coefficient is much larger than its absorption coefficient. Ratio of radiation intensity from reduced scaling plume to that of the full scaled one increases with the scaling ratio. The power of the growth curve is 1.5 for azimuth angle $\theta=0^{\circ}$, becomes 2 for $\theta=40^{\circ}$, and arrives at 2.5 for $\theta=120^{\circ}$ and $160^{\circ}$. There is good similarity between spectral variations of plumes from different scaling solid rockets, and there are two peak spectra of radiation intensity in $2.7 \sim 3.0 \mu \mathrm{m}$ and $4.3 \sim 4.6 \mu \mathrm{m}$. Radiation intensity of the plume in rear hemisphere is bigger than that in the front, and the effect of radiance from the rear end surface makes the direction with maximum radiation intensity deflects toward the rear hemisphere.

\section{Conflict of Interests}

There is not conflict of interests for all authors of this paper. 


\section{Acknowledgments}

This work is cosponsored by the National Natural Science Foundation of China (51376065, 51176052), Guangdong Key Scientific Project (2013B010405004), Guangdong Province Key Laboratory of Efficient and Clean Energy Utilization (2013A061401005), South China University of Technology, and Key Laboratory of Efficient and Clean Energy Utilization of Guangdong Higher Education Institutes (KLB10004).

\section{References}

[1] G. L. Wang, "Design of solid rocket engine," chapter 3, Publishing House of Northwest University, Xi'an, China, 1994.

[2] V. V. Rozanov and A. I. Lyapustin, "Similarity of radiative transfer equation: error analysis of phase function truncation techniques," Journal of Quantitative Spectroscopy and Radiative Transfer, vol. 111, no. 12-13, pp. 1964-1979, 2010.

[3] Z. Jun and P. Wenbing, "Similarity transformations of radiation hydrodynamic equations and investigation on laws of radiative conduction," Physics of Fluids B, vol. 4, no. 4, pp. 872-876, 1992.

[4] T. Duracz and N. J. Mccormick, "Equations for estimating the similarity parameter from radiation measurements within weakly absorbing optically thick clouds," Journal of the Atmospheric Sciences, vol. 43, no. 5, pp. 486-492, 1986.

[5] C. Mitrescu and G. L. Stephens, "On similarity and scaling of the radiative transfer equation," Journal of Quantitative Spectroscopy and Radiative Transfer, vol. 86, no. 4, pp. 387-394, 2004.

[6] A. I. Bril, V. P. Kabashnikov, N. V. Kuzmina, and V. M. Popov, "Similarity of heat radiation from turbulent buoyant jets," International Journal of Heat and Mass Transfer, vol. 41, no. 10, pp. 1347-1356, 1998.

[7] D. K. Edwards, "Molecular gas band radiation," Advances in Heat Transfer, vol. 12, pp. 115-193, 1976.

[8] W. Malkmus, "Random Lorentz band model with exponentially tailed $\mathrm{S}^{-1}$ line intensity distribution function," Journal of the Optical Society of America, vol. 57, no. 3, p. 323, 1967.

[9] J. M. Hartmann, R. Levi Di Leon, and J. Taine, "Line-by-line and narrow-band statistical model calculations for $\mathrm{H}_{2} \mathrm{O}$," Journal of Quantitative Spectroscopy and Radiative Transfer, vol. 32, no. 2, pp. 119-127, 1984.

[10] M. F. Modest, "Weighted-sum-of-gray-gases model for arbitrary solution methods in radiative transfer," Journal of Heat Transfer, vol. 113, no. 3, pp. 650-656, 1991.

[11] R. A. Reed and V. S. Calia, "Review of aluminum oxide rocket exhaust particles," in Proceedings of the 28th AIAA Thermophysics Conference, AIAA-93-2819, Orlando, Fla, USA, July 1993.

[12] R. A. Reed and V. S. Calia, "New measurements of liquid aluminum oxide," in Proceedings of the JANNAF Exhaust Plume Subcommittee Meeting, Philips Laboratory, Kirland AFB, Albuquerque, NM, USA, 1993.

[13] C. F. Bohran and D. R. Huffman, Absorption and Scattering of Light by Small Particles, John Wiley \& Sons, New York, NY, USA, 1983.

[14] Q. Yu, L. Liu, Y. Pan, D. Zhang, J. Ji, and H. Tan, "Monte Carlo method for simulating the radiative characteristics of an anisotropic medium," Heat Transfer: Asian Research, vol. 28, no. 3, pp. 201-210, 1999.
[15] D. A. Blank and S. C. Mishra, "Use of the 2-D collapsed dimension method in gray enclosures with absorbing-emittingisotropic scattering media in radiative equilibrium," Numerical Heat Transfer Part B, vol. 30, no. 4, pp. 469-481, 1996.

[16] N. Selçuk and N. Kayakol, "Evaluation of discrete ordinales method for radiative transfer in rectangular furnaces," International Journal of Heat and Mass Transfer, vol. 40, no. 2, pp. 213222, 1997.

[17] E. H. Chui, G. D. Raithby, and P. M. J. Hughes, "Prediction of radiative transfer in cylindrical enclosures with the finite volume method," Journal of Thermophysics and Heat Transfer, vol. 6, no. 4, pp. 605-611, 1992.

[18] Z. Li, H.-J. Xiang, and X.-Y. Zhang, "Numerical simulation of composite solid propellant rocket motor exhaust plume," Journal of Solid Rocket Technology, vol. 37, no. 1, pp. 37-42, 2014.

[19] P. C. Braithwaite, "Quench bomb investigation of aluminum oxide formation from solid rocket propellants (part I): experimental methodology," in Proceedings of the 25th JANNAF Combustion Meeting, CPIA Publication 498, 1988.

[20] S.-W. Fan, X.-Y. Zhang, D.-Q. Zhu, H.-J. Xiang, and G.-B. Cai, "Calculation of the infrared characteristics of the solid rocket plume with FVM method," Journal of Astronautics, vol. 26, no. 6, pp. 793-797, 2005.

[21] C. B. Ludwig, W. Malkmus, J. E. Reardon et al., Handbook of Infrared Radiation from Combustion Gases, NASA-SP-3080, Hamppon Virainiaz Lanzley Research Center, 1973.

[22] D. Q. Zhu, X. Y. Zhang, H. J. Xiang et al., "Cooling techniques for high pressure chamber of LOX/kerosene engine," Journal of Astronautics, vol. 29, no. 1, pp. 255-259, 2008. 


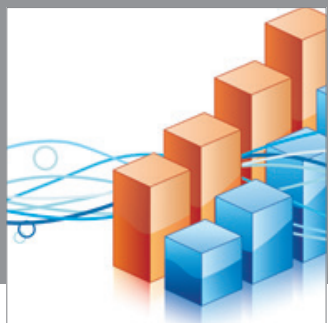

Advances in

Operations Research

mansans

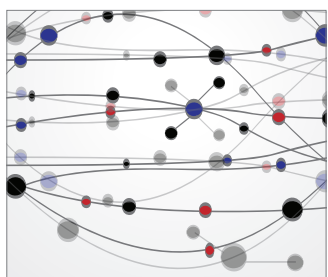

The Scientific World Journal
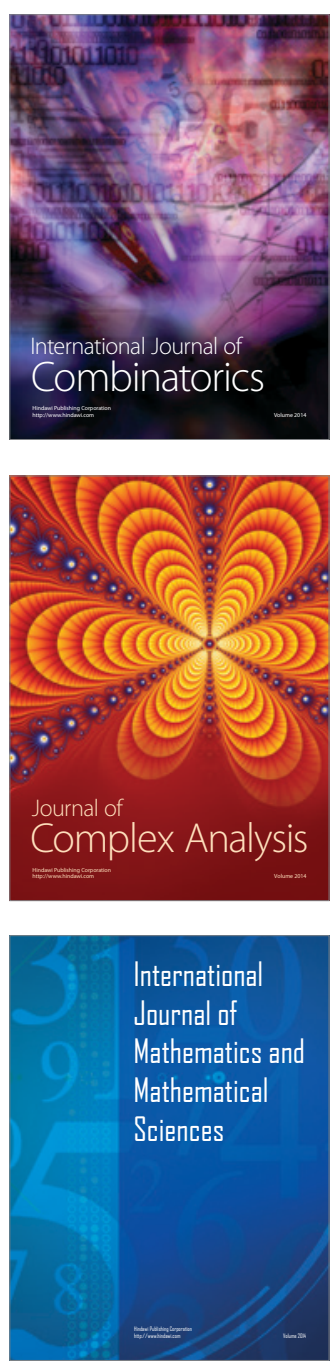
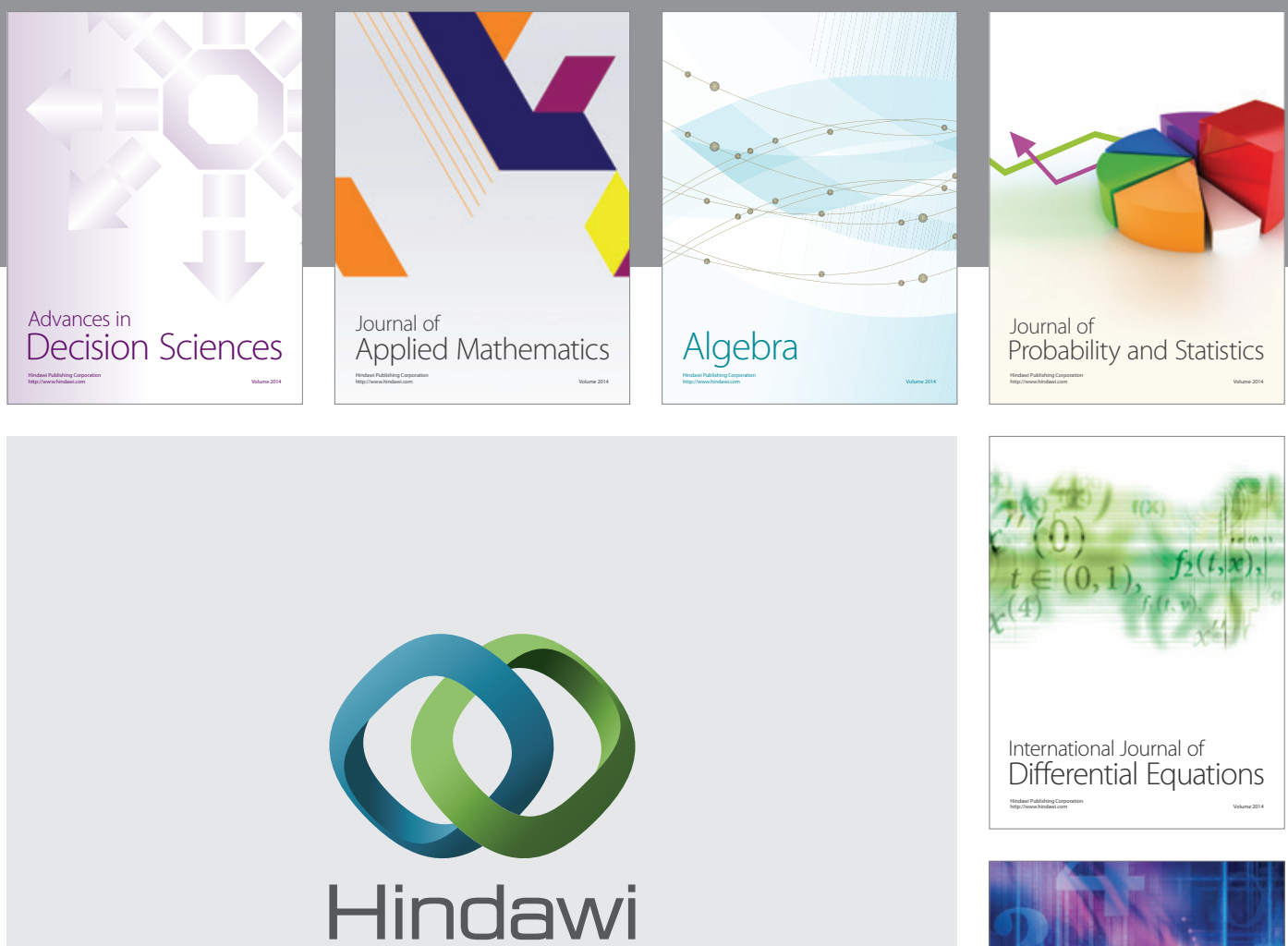

Submit your manuscripts at http://www.hindawi.com
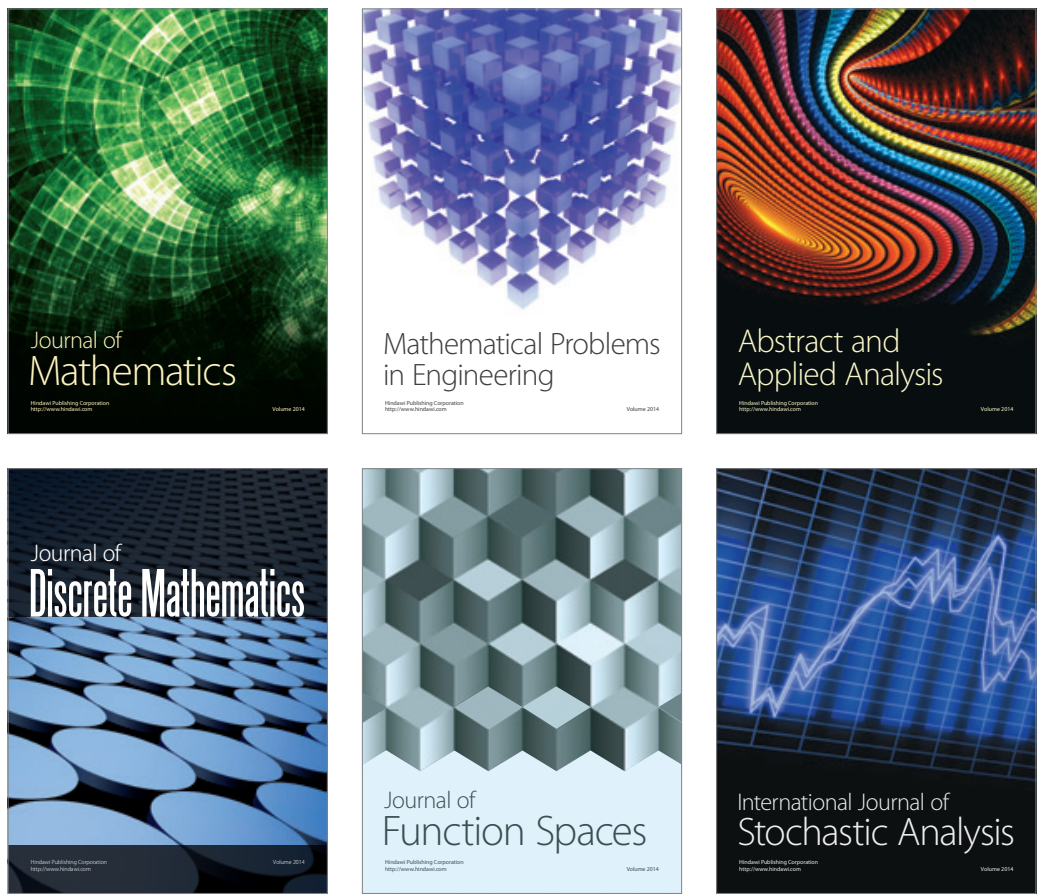

Journal of

Function Spaces

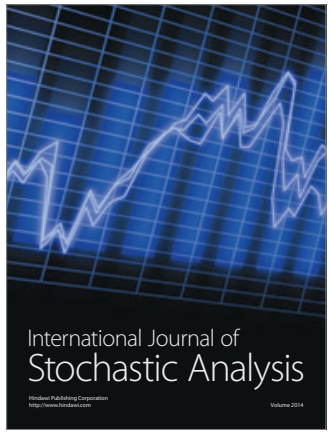

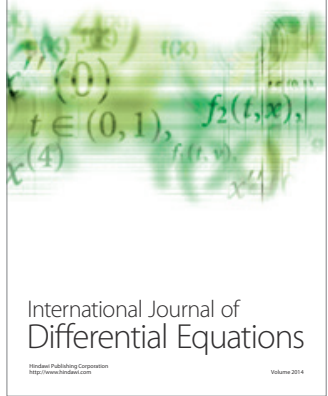
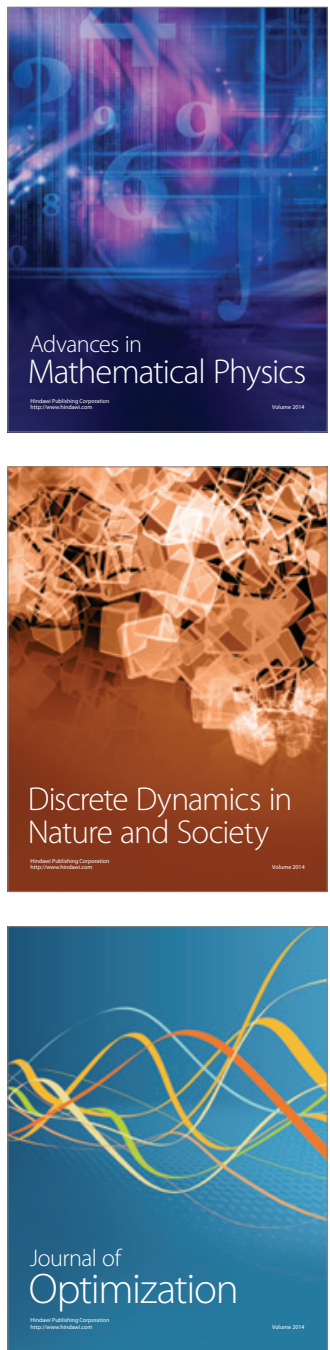